\title{
Assessing decision strategies using HyperCard
}

\author{
MELISSA L. FINUCANE, DAN MILECH, and MURRAY T. MAYBERY \\ University of Westerm Australia, Nedlands, Australia
}

\begin{abstract}
The use of "process-tracing" techniques for examining individuals' decision strategies has been criticized widely for yielding results that are uninformative and often confounded. The present paper describes a new technique based on a "structural" approach that allows efficient assessment of finely discriminated decision strategies via a HyperCard program called DECOUT. The program presents individuals with an information matrix (e.g., describing five alternative pesticides on three attributes). Individuals are asked to choose the best or the worst alternative in the matrix for 12 problems. Information in the matrix is constructed so that choice of an alternative by an individual is uniquely associated with use of one particular decision strategy. The strategies include information integration, maximin, maximax, pessimism-optimism, and minimax-regret processes. An experiment using the program is described, and possible directions for future research and development are discussed.
\end{abstract}

A long-standing problem for researchers in decision making has been the lack of an efficient and accurate technique for assessing decision strategies. We have developed a new technique whereby minimal data can be used in order to distinguish among specific decision strategies. Our technique revitalizes the seemingly forsaken "structural" approach in decision-making research, insofar as it estimates an individual's decision strategy on the basis of his/her decision outcome. The main feature of our technique is that we can provide individuals with decision problem information in such a way that their preferred option (alternative) in the problem is uniquely associated with one of five specific decision strategies.

Our technique has both methodological and theoretical value. In this paper, we focus on the methodological value first. We then describe an example of a theoretical question that can be examined by using the technique. We report an experiment in which the technique was used, and finally, we discuss possible directions for future research and development.

\section{METHODOLOGICAL VALUE}

Our technique is an alternative to the recently popular "process-tracing" techniques. Process-tracing typically focuses on various parameters of individuals' information search and acquisition strategies, such as the order in which information is retrieved and the duration of access to information (for reviews, see Ford, Schmitt, Schechtman, Hults, \& Doherty, 1989; Harte, Westenberg, \& van

The authors gratefully acknowledge Sylvie Noël for her generous help with programming the experimental stimuli. The DECOUT program will run on Apple Macintosh computers with the application HyperCard; it can be obtained by sending a blank 3.5-in. Apple-formatted disk to M. Finucane, Department of Psychology, University of Western Australia, Nedlands, WA 6907, Australia. Other correspondence can be sent to the same address. E-mail should be sent to melissa(a)psy.uwa. edu.au.
Someren, 1994). The process-tracing approach has several methodological difficulties, however, which potentially lead to data that are uninformative and confounded. The first difficulty is that generalizability may be limited. For example, some studies have involved close observation of participants' information search strategies through videotaping or audiotaping (see Biggs, Rosman, \& Sergenian, 1993). Demand characteristics influence such tasks, since participants are aware that their decision processes are being observed and may arbitrarily alter their information acquisition strategies to confirm the experimenter's suspected hypothesis. A second difficulty is the potential introduction of biases in an individual's search pattern due to response biases that can occur as that individual reads the information that is presented. People prefer, for example, to read from left to right and from top to bottom. Third, process-tracing tends to focus on what information is searched rather than how the information is processed. Fourth, process-tracing techniques are inefficient, often requiring a substantial amount of data and needing at least two independent raters to decode the data into particular search strategy types. Finally, individuals' responses may be decoded unreliably and possibly only at a general level (such as the broad categories of "compensatory" and "noncompensatory").

The problems above were avoided by programming our new technique in a HyperCard stack called DECOUT (Decision Outcome). Data about individuals' decisionmaking strategies are collected unobtrusively by the computer, making it less likely that participants will guess the experimenter's interest in decision strategies. Potential bias from reading preferences is minimized by having participants familiarize themselves with the attributes prior to exposure to the information board, by counterbalancing the presentation of information given for options and attributes, and by having all information in the board available to participants from the start of the decision problem. Only a very small amount of data is required for analysis, and it is easy to discriminate among decision strate- 
gies at a specific level. Because of the mathematically precise nature of the information board, we avoid subjective codings of types of search strategies in participants' responses. Rather, decision strategies are inferred from the more objective measure of decision outcomes.

Essentially, the technique presents individuals with an information board describing five options $\left(\mathrm{O}_{1}-\mathrm{O}_{5}\right)$ on three attributes $\left(a_{1}-a_{3}\right)$. Values in the board are specified by the researcher in such a way that a participant's preference for an option is uniquely related to one of five decision-making strategies (described in the next section). Traditionally, decision-making researchers illustrated decision problems in payoff matrix form. However, for the purposes of our research, the payoff matrix is being applied in a new way: rather than as a way of evaluating actions and outcomes under given states of nature, the matrix is used for evaluating information in terms of options. Thus, the evaluation of an option $\left(O_{i}\right)$ from among options $O_{1}, \ldots, O_{n}$, in respect of an attribute $\left(a_{j}\right)$ from among attributes $a_{1}, \ldots, a_{m}$, results in a value reflected by the entries $v_{i j}$ (see Figure 1; and for a more concrete illustration, see Figure 3).

We view the technique as a "structural modeling approach," insofar as it estimates an individual's decision strategy on the basis of his/her decision outcome and describes the decision strategies with algebraic models. Of crucial importance in the technique are the stimuli in the information board, the importance individuals place on different attributes, and individuals' decision responses, as they form the basis for the researcher's inferences about the decision strategies used by individuals.

\section{THEORETICAL VALUE}

The theoretical importance of our technique is that different theories of decision making can be compared directly. How people combine information when making decisions has been researched for several decades, but a clear comparison of theories has been difficult to obtain. We were interested in using DECOUT to compare two decision-making theories: information integration theory (Anderson, 1974, 1990) and what we call simplification theory, which has been proposed in different forms by several researchers (e.g., Hurwicz, 1951; Milnor, 1954;
Savage, 1951). Specifically, we wanted to know whether we could assess individuals as using an information integration decision strategy, or one of several simplification strategies, for a decision task presented via DECOUT.

According to information integration theory, people are "intuitive statisticians" who integrate all available information when making evaluations. The theory emphasizes the particular rules (i.e., cognitive algebra) that individuals use to integrate information and form intuitive estimates (Busemeyer, 1990). The rules most reported by researchers include adding, averaging, and multiplying processes. Information integration is modeled with equations in which the parameters are determined subjectively rather than objectively. One of the most straightforward and comprehensive models reported by researchers is the "linear model." This model is based on the assumption that when evaluating an option characterized on several attributes, an individual measures the extent that each attribute is represented in the option (implicitly, at least), and gives the attribute a weight reflecting its relative importance. The evaluation of each option is the sum of the weighted attribute values:

value of option $=$

sum of (relative weight $\times$ scale value) of all attributes.

Repeating this process for the other options, an individual then chooses the option having the greatest value. According to the linear model, an individual considers explicitly all the information concerning the options and assigns weights to each attribute, which, given the algebraic form of the model, reflect the extent to which one attribute will be "traded off" against another (Hogarth, 1980).

For example, a gardener might be deciding which pesticide to use on vegetables. The options being evaluated are the different pesticides available. Two attributes characterizing the pesticides are their specificity (in targeting a particular pest) and effectiveness. When evaluating each pesticide, the gardener may weight the specificity of the pesticide as more important than its effectiveness. The value of a pesticide is determined by summing (1) the product of the relative weight and value for the specificity with (2) the product of the relative weight and value for the effectiveness. The best choice for the gardener will be the pesticide with the greatest outcome value. By trading

\begin{tabular}{|c|c|c|c|c|}
\hline & \multicolumn{3}{|c|}{ Attributes } \\
\hline & & $\mathbf{a}_{1}$ & $\mathbf{a}_{2}$ & $a_{3}$ \\
\hline \multirow{5}{*}{ Options } & $\mathrm{O}_{1}$ & 8 & 0 & 8 \\
\hline & $\overline{\mathrm{O}_{2}}$ & 5 & 5 & 5 \\
\hline & $\overline{\mathrm{O}_{3}}$ & 10 & $\overline{0}$ & 0 \\
\hline & $\overline{\mathbf{O}_{4}}$ & 9 & 2 & 2 \\
\hline & $\overline{\mathbf{O}_{5}}$ & 6 & 1 & 4 \\
\hline
\end{tabular}

Decision strategy and option selected by it

Information Integration
Maximin
Maximax
Pesimisn-Optimism $(\alpha=1 / 2)$
Minimax-Regret

Figure 1. An example of the values presented in a decision problem where five different options are described on three attributes. Note that the problem is constructed so that each decision strategy uniquely selects a "most preferred" option, and these selections are given in the final column. 
off specificity against effectiveness, the gardener may choose a pesticide with low effectiveness but high specificity, over a pesticide that has high effectiveness but low specificity.

The linear model has been cited by many as well suited for preference judgments. One advantage is that when there are several options to consider in an evaluation, the model handles all the information in a consistent way. The linear model has been shown to be remarkably accurate in predicting individual evaluations in both laboratory and field situations. Several researchers have reported, however, that the linear model is inadequate as a description of evaluation processes. The model implies a process of explicit calculations and the trading off of attributes which is infeasible when there are many attributes and options to consider. Furthermore, there is evidence that this process is avoided even when the number of attributes and options is small (Einhorn, Kleinmuntz, \& Kleinmuntz, 1979; Hogarth, 1980).

In contrast to information integration theory, in which individuals are thought to consider all information available when they make evaluations, several researchers have proposed that individuals may reduce cognitive processing by considering only some information. That is, individuals are thought to use simplification strategies when they make evaluations. There has been considerable debate, however, about exactly how this simplification occurs. In general, researchers suggest that people rely on the principle of "divide and conquer" (Slovic, Fischhoff, \& Lichtenstein, 1977, p. 17). That is, the decision maker is thought to partition the total problem into a series of structurally related parts and then assess only the smallest components. Theorists argue that simplification processes in decision making are frequently effective and lead to evaluations that are accurate in many cases (Slovic et al., 1977).

Several theories have been proposed which describe simplification strategies; each theory gives a precise description of the strategy which is used in simplifying a problem when one is making an overall evaluation. One of the most simple, clear, and complete descriptions of these theories has been presented by Coombs, Dawes, and Tversky (1970), who describe the various simplification processes that may be used by individuals making evaluations. Other descriptions of simplification processes are typically subsets of this (and are usually ad hoc). Four simplification strategies described by Coombs et al. will be outlined in this section: maximin, maximax, pessimismoptimism, and minimax-regret.

\section{The Maximin (Wald) Strategy}

This is one of the simplest strategies, whereby individuals choose an option that maximizes the minimum attribute value, regardless of the attributes available for evaluation. That is, each option is evaluated by comparison with the worst case possible. This strategy is optimal if the worst that could happen always happens. Coombs et al. (1970) have called the strategy conservative and pessimistic, and they have suggested that it is best used in contexts in which extreme caution is desired.

\section{The Maximax (Complete Optimism) Strategy}

This is also a very simple strategy in which individuals choose an option whose highest attribute value is maximal. That is, each option is evaluated by comparison with the best case possible. This strategy is optimal if the best that can happen always happens.

\section{The Pessimism-Optimism (Hurwicz) Strategy}

This strategy, which generalizes the maximin and the maximax processes, is used when individuals make decisions on the basis of the largest and smallest attribute standings for each option and ignore the other standings. This process is represented mathematically by the individual choosing the option with the highest value from a pessimism-optimism index. The value for each option $O_{i}$ is defined as $\alpha v_{i}^{\prime}+(1-\alpha) v_{i}^{\prime \prime}$, where $0<\alpha<1$, and $v_{i}^{\prime}$ and $v_{i}^{\prime \prime}$ denote the option's minimum and maximum attribute values, respectively.

\section{The Minimax-Regret (Savage) Strategy}

In this strategy, individuals assign each option a maximal regret value for each attribute, which is the difference between the attribute value for the option, and the maximal value among all options on that attribute. Individuals then choose an option that has minimal maximal regret. This process is similar to the maximin strategy, since the focus is on the worst possible option, but "worst" is defined by maximal regret rather than minimum attribute value.

Returning to the example of the gardener's choice of pesticides, the maximin strategy would consist of finding the lower value for each pesticide on the attributes of specificity and effectiveness and then choosing the pesticide with the highest of these minimum values. Maximax would consist of choosing the pesticide with the highest value on either specificity or effectiveness. As indicated above, the pessimism-optimism and minimaxregret strategies would use the minimum and maximum attribute values in different ways.

Although these simplification strategies were proposed by researchers some time ago, investigations have been plagued with methodological problems, and little light has been shed on individuals' use of the strategies. Decision time has been the preferred indicator of individuals' use of simplification strategies, making it impossible for researchers to determine whether individuals actually use such strategies, whether a single preexisting strategy is modified according to the circumstances, or whether alternative strategies are used in different circumstances. Thus, individual differences in the use of qualitatively different simplification strategies need investigation. In particular, the use of different strategies needs to be distinguished from the use of different parameters for a single invariant strategy. We expected that DECOUT would allow us to investigate individuals' use 
of various simplification strategies without falling prey to the methodological difficulties experienced by previous researchers. We also expected to compare directly the extent to which individuals opted for the information integration strategy versus a simplification strategy when making decisions.

\section{DESCRIPTION OF AN EXPERIMENT USING DECOUT}

The aim of the experiment was to determine whether different decision strategies were used when individuals made decisions on the basis of either positive or negative information given about different hazards (pesticides or medical x-rays). Seventy-two participants (mean age, 20.3 years) were recruited from undergraduate psychology classes and given course credit for their participation.

In the experiment, DECOUT comprised four critical sections: (1) the introduction, which defined the hazard of interest (e.g., pesticides) and the attributes (e.g., negative effects on physical health) used in the information board; (2) the attribute rating task, which identified the relative importance that a participant assigned to each attribute; (3) the decision problems, which included 2 examples, 4 practice problems, and 12 experimental problems; and (4) the hidden workings, which contained cards inaccessible to participants but used to change various parameters of the stack, including the hazard, the attributes, and the problem values presented to participants.

Special external commands and functions were used to create Microsoft Excel files in which participants' responses were recorded as they progressed through DECOUT. HyperTalk scripts were used for all other processes in the stack, such as controlling navigation through the stack, and adjusting values in the decision problems to reflect individuals' relative importance attribute ratings.

To expand on Section 2 of DECOUT, the attribute rating task, we note one important assumption of our approach: that each individual weights the attribute values according to the relative importance of the attributes. The board in Figure 1 would allow the researcher to discriminate among the five decision strategies for an individual who assigned equal importance to the attributes, but the values would have to be adjusted for an individual who assigned differential importance to the attributes. These adjustments were made using the information from the attribute rating task, which was done once before the example problems, and once before the set of experimental decision problems.

In the attribute rating task, participants were shown all three attributes and were asked to choose the one they considered least important by clicking on it with the mouse. When a choice was made, DECOUT instructed participants that the chosen attribute was assigned a rating of "10," and this rating appeared on the left of the attribute. Participants were then instructed to assign importance ratings to the remaining attributes relative to the least important attribute. For example, if a participant thought an attribute was twice as important as the least important attribute, it should be given a rating of "20." Participants indicated their ratings by typing the relevant number in a designated box on the left of each attribute (see Figure 2).

DECOUT used the relative ratings to adjust the attribute values used in the decision problems. For example, if the rating task showed the relative importance to be $2: 1: 1$ for attributes $a_{1}, a_{2}$, and $a_{3}$, then a suitable adjustment to the attribute values would be to halve the range of values for

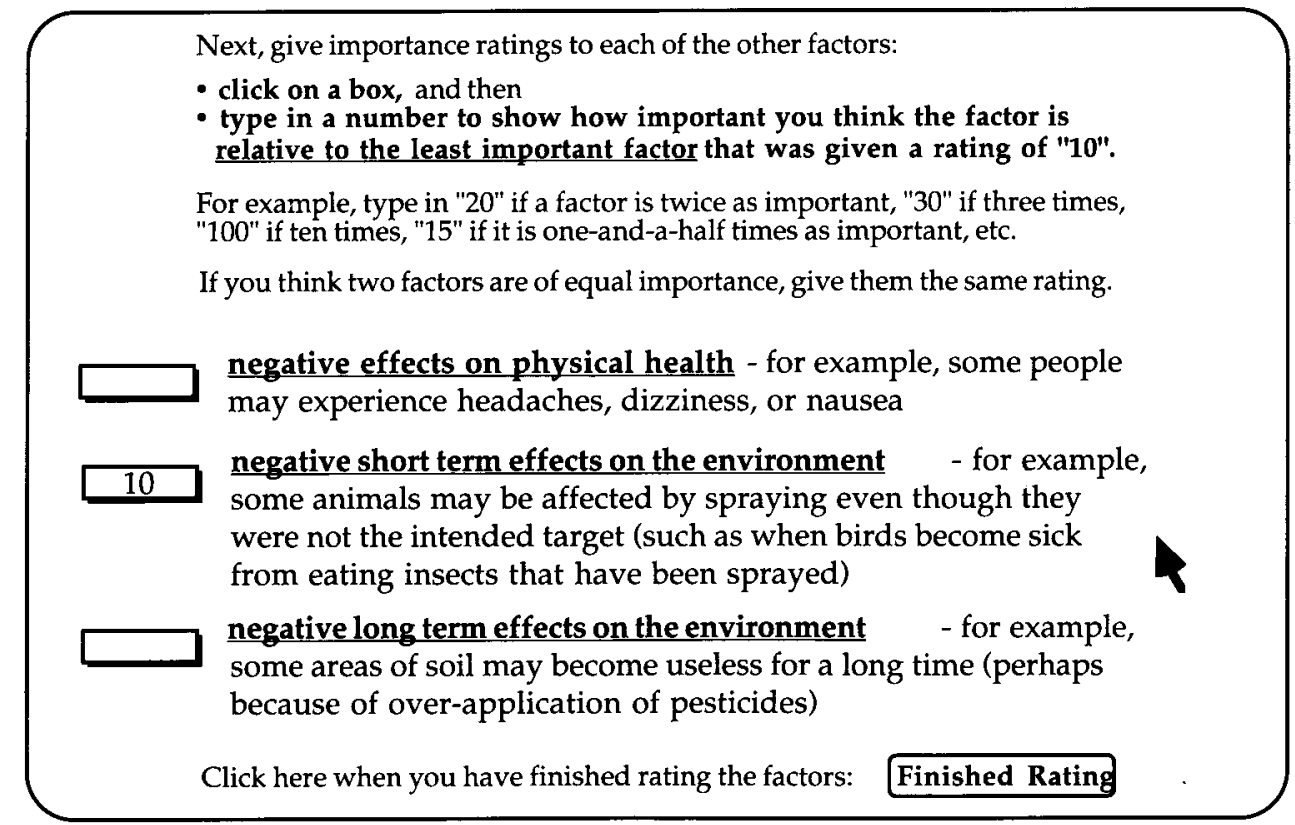

Figure 2. Example of the attribute rating task presented by DECOUT in which participants are asked to indicate the relative importance of each attribute. 
$a_{1}$ (e.g., values of $4,2.5,5,4.5$, and 3 for the board in Figure 1).

For Section 3 of DECOUT, the decision problems, we used two basic information boards that conformed to the mathematical requirements shown in Figure 1. From the boards, we constructed 12 decision problems that looked superficially different from each other, but essentially presented information so that choice of one option in a decision problem was uniquely associated with one decision strategy. Six of the problems were used as the example and practice problems, and the full set of 12 was used as the experimental problems. Participants were asked to indicate the worst option when given negative information, or the best option when given positive information. Participants responded by clicking with a mouse on the letter that corresponded to the option that they wanted to choose (for an example, see Figure 3 ).

Comparison of Figures 1 and 3 shows that the attribute values used are the same in these illustrations. Hence, choosing the first option in the decision problem shown in Figure 3 would indicate use of the information integration strategy (as specified in Figure 1); choosing Pesticide $\mathrm{B}$ would indicate use of the maximin strategy; choosing Pesticide $\mathrm{C}$ would indicate use of the maximax strategy; and so on.

Each participant worked through DECOUT at his/her own pace. At the end, a brief interview was conducted in which each participant was asked to "think aloud" while working through the first two decision problems again.

We calculated the percentage of experimental problems for which each option, corresponding to each of the five decision strategies, was chosen by a participant. According to the binomial distribution, one particular strategy needed to be endorsed consistently by a participant for at least 6 out of the 12 trials in order for us to reject the hypothesis (at $\alpha=.05$ ) that random choices were being made. Results indicated that $44.4 \%$ of participants could be assessed as using one of two choice strategies: information integration or maximin. None of the participants was assessed as using the maximax, pessimismoptimism, or minimax-regret strategy. Yates's corrected Pearson chi-squares were calculated to determine whether the information valency (positive vs. negative) or hazard type (pesticides vs. $x$-rays) affected the frequency with which a strategy was evidenced. A significant effect was found for information valency $\left[\chi^{2}(2)=13.4, p<.01\right]$. The pattern of responses indicated that there was a tendency for a higher proportion of participants to use information integration when presented with negative information $(23.6 \%)$ than when presented with positive information $(5.6 \%)$, but for a higher proportion of participants to use maximin when presented with positive information $(12.5 \%)$ than when presented with negative information $(2.8 \%)$. There was no significant effect of hazard type, and the interaction between information valency and hazard type was not significant.

Responses by participants in the postexperimental interviews indicated that participants who were assessed as using one decision strategy consistently tended to articulate the appropriate information integration or maximin strategy. Responses of other participants provided reasons why they might not have been assessed as using one of the five expected decision strategies. First, some participants appeared to use a strategy that was not included in the five that we set up to be uniquely predicted by the decision problems. Second, some participants seemed to vary their attribute importance weights or their decision strategies across the experimental problems.

\section{PROBLEM1}

Examine the pesticides below and pick out the worst.

\begin{tabular}{|c|c|c|c|c|}
\hline & $\begin{array}{l}\text { Negative } \\
\text { effects on } \\
\text { physical } \\
\text { health }\end{array}$ & \begin{tabular}{|c|} 
Negative \\
short term \\
effects on the \\
environment
\end{tabular} & \begin{tabular}{|c|} 
Negative \\
long term \\
effects on the \\
environment
\end{tabular} & $\begin{array}{l}\text { Which pesticide is worst? } \\
\text { (Answer by clicking on one } \\
\text { letter). }\end{array}$ \\
\hline Pesticide A & 8.00 & 0.00 & 8.00 & A \\
\hline Pesticide B & 5.00 & 5.00 & 5.00 & $B$ \\
\hline Pesticide C & 10.00 & 0.00 & 0.00 & C \\
\hline Pesticide D & 9.00 & 2.00 & 2.00 & $\mathrm{D}$ \\
\hline Pesticide E & 6.00 & 1.00 & 4.00 & $E$ \\
\hline
\end{tabular}

Figure 3. Example of a decision problem presented by DECOUT in which participants are asked to indicate which is the worst option. 
Scrutiny of strategies that participants reported - other than those we were expecting-indicated that the most popular seemed to be a variant of the information integration strategy. Instead of considering information for all three attributes, participants selected only the two attributes that they considered most important; then they combined these attributes' values (using an additive linear model) and chose the option with the highest outcome value. The variant strategy can be identified from the 12 decision problems that we used. However, the options associated with the strategy differ, depending on which two columns of values are being compared. For example, for the information board shown in Figure 1, a participant focusing on attributes $a_{1}$ and $a_{2}$ would choose option $O_{4}$, a participant focusing on attributes $a_{2}$ and $a_{3}$ would choose option $\mathrm{O}_{2}$, and a participant focusing on attributes $a_{1}$ and $a_{3}$ would choose option $O_{1}$. Fortunately, the sequence of decision outcomes across problems for a participant using a variant strategy is different from the sequence found when one of the originally expected strategies is used, owing to counterbalancing of the assignment of values to attributes across the 12 problems for each subject. Thus, we could distinguish participants who used a variant strategy from those using the other strategies. Our post hoc analyses of the decision data indicated that $45.8 \%$ of the participants could be assessed as using one of the information integration variants.

The variation of the participants' attribute importance weights across the experimental problems could not be assessed in the current version of DECOUT. However, a future version could easily be designed to elicit information from participants that would allow patterns of weight shifting to be detected from problem to problem.

\section{CONCLUSIONS AND SUGGESTIONS FOR FUTURE RESEARCH}

Overall, the experiment demonstrated that our new technique for assessing decision strategies via DECOUT maximizes research and analysis potential in the field of decision making, and that it minimizes the time and effort required in order to collect and analyze decision strategy data. Our technique addressed several methodological pitfalls common in process-tracing techniques and facilitated direct comparison of theories about the type of decision strategies that individuals might use to choose among options. The experimental task took approximately 30 min per participant, and data collection and analysis were made manageable by the automatic recording of participant responses by DECOUT. The use of HyperCard to develop DECOUT demonstrated an inexpensive but reliable technology for collecting data from controlled experimental conditions. Given the simplicity of the concepts and interface in DECOUT, it can easily be applied to various decision problems.

One of the main criticisms of using a structural approach in decision research is that data are gathered typically after a decision has been made, whereas in process-tracing studies, data are gathered during the decision process
(Westenberg \& Koele, 1994). A modified version of DECOUT could incorporate process-tracing techniques for empirical comparison of the two approaches to assessing decision strategies. For example, future development might include a "peek-a-boo" feature in which values in the information board are hidden unless requested by participants, and DECOUT could record the order and duration as participants search the information.

Finally, future research might concentrate on adding options to the information board to identify other decision strategies, such as the information integration variant. Such a development would be simple with DECOUT, because alterations to the information board values can be made easily in the hidden workings (Section 4) of the program, which allows parameters to be changed directly by the researcher. The incorporation of any new strategy into the technique obviously needs to conform to the mathematical requirements that allow decision strategies to be estimated from decision outcomes. Fortunately, in subsequent research, we have found this process to be straightforward.

\section{REFERENCES}

ANDERSON, N. H. (1974). Information integration theory: A bref survey. In D. H. Krantz, R. C. Atkinson, R. D. Luce, \& P. Suppes (Eds.), Contemporary developments in mathematical psychology (Vol. 2, pp. 236-305). San Francisco: W. H. Freeman.

ANDERSON, N. H. (1990). A cognitive theory of judgment and decision. In N. H. Anderson (Ed.), Contributions to information integration theory (Vol. 1, pp. 105-142). Hillsdale, NJ: Erlbaum.

Biggs, S. F., Rosman, A. J., \& Sergenian, G. K. (1993). Methodological issues in judgment and decision-making research: Concurrent verbal protocol validity and simultaneous traces of processes. Journal of Behavioral Decision Making, 6, 187-206.

BUSEMEYER, J. R. (1990). Intuitive statistical estimation. In N. H. Anderson (Ed.), Contributions to information integration theory (Vol. 1 , pp. 187-215). Hillsdale, NJ: Erlbaum.

CoOmBs, C. H., DaWes, R. M., \& TVERSKY, A. (1970). Mathematical psychology: An elementary introduction. Englewood Cliffs, NJ: Prentice-Hall.

EinhoRn, H. J., KLEINMUNTZ, D. N., \& KLEINMUNTZ, B. (1979). Linear regression and process tracing models of judgment. Psychological Review, 86, 465-485.

Ford, J. K., Schmitt, N., Schechtman, S. L., Hults, B. M., \& DoHERTY, M. L. (1989). Process tracing methods: Contributions, problems, and neglected research questions. Organizational Behavior \& Human Decision Processes, 43, 75-117.

Harte, J. M., Westenberg, M. R. M., \& van Someren, M. (1994). Process models of decision making. Acta Psychologica, 87, 95-120.

HogARTH, R. M. (1980). Judgement and choice: The psychology of decision. New York: Wiley.

HuRWICZ, L. (1951). Some specification problems and application in econometric models. Econometrica, 19, 343-344.

MiLNOR, J. (1954). Games against nature. In R. M. Thrall, C. H. Coombs, \& R. L. Davis (Eds.), Decision processes (pp. 49-59). New York: Wiley.

SAVAGE, L. J. (1951). The foundations of statistics. New York: Wiley.

SLOvic, P., FischHoff, B., \& Lichtenstein, S. (1977). Behavioral decision theory. Annual Review of Psychology, 28, 1-39.

Westenberg, M. R. M., \& Koele, P. (1994). Multi-attribute evaluation processes: Methodological and conceptual issues. Acta Psychologica, 87, 65-84.

(Manuscript received November 13, 1995; accepted for publication December 20,1995.) 